Supporting Information for

\title{
Electrochemical imaging of endothelial permeability using a large-scale integration-based device
}

Kosuke Ino ${ }^{1, *}$, Hao-Jen $\mathrm{Pai}^{2}$, Kaoru Hiramoto ${ }^{2}$, Yoshinobu Utagawa ${ }^{2}$, Yuji Nashimoto ${ }^{1,3}$, Hitoshi Shiku ${ }^{1, *}$

${ }^{1}$ Graduate School of Engineering, Tohoku University, 6-6-11 Aramaki-aza Aoba, Aobaku, Sendai 980-8579, Japan

${ }^{2}$ Graduate School of Environmental Studies, Tohoku University, 6-6-11 Aramaki-aza Aoba, Aoba-ku, Sendai 980-8579, Japan

${ }^{3}$ Frontier Research Institute for Interdisciplinary Sciences, Tohoku University, 6-3 Aramaki-aza Aoba, Aoba-ku, Sendai 980-8578, Japan

Corresponding authors: Kosuke Ino (kosuke.ino@tohoku.ac.jp) and Hitoshi Shiku (hitoshi.shiku.c3@tohoku.ac.jp)

\section{Table of Contents}

- Figure S1

- Figure S2

- Figure S3 
(A)

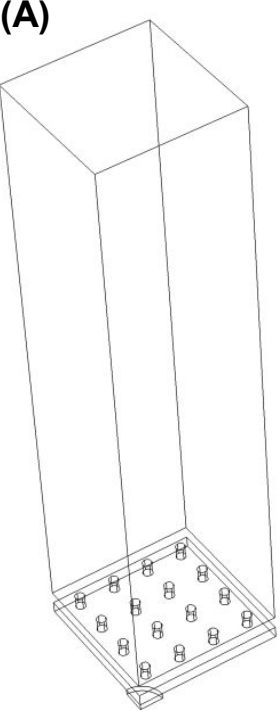

(B)

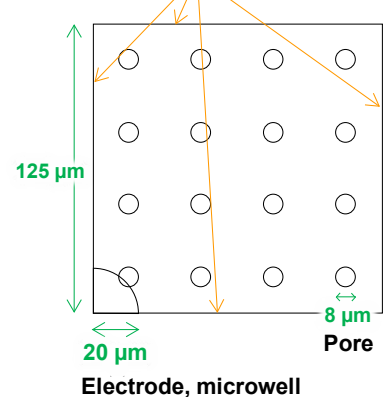

(C) Boundary conditions: $5 \mathrm{mM}$ Bulk $\mu \mathrm{m}$

Initial values: $5 \mathrm{mM}$

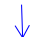

$\uparrow 1,5,10,20$, or $50 \mu \mathrm{m}$ Gap between the device and the membrane

Boundary conditions: $0 \mathrm{mM}$

Electrode, electrochemical reaction

(D)

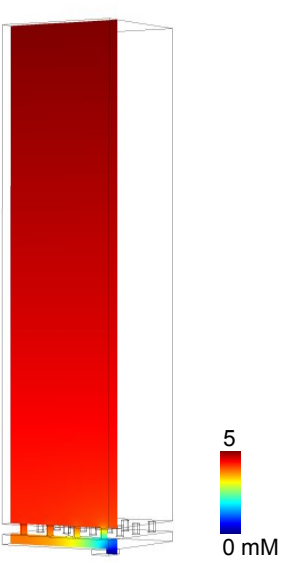

(E)

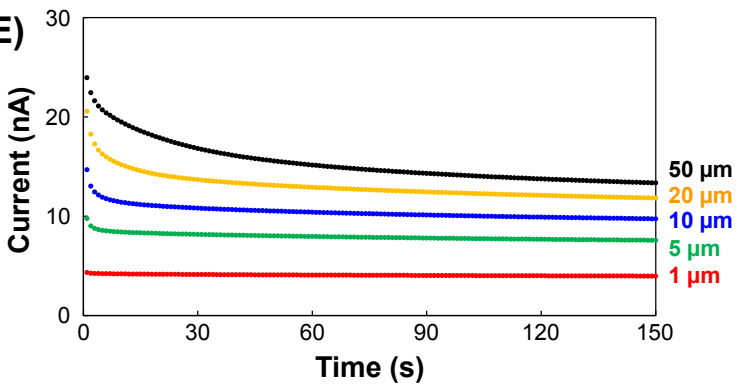

\section{Figure S1}

Current simulation for redox compound diffusion using COMSOL Multiphysics (ver. 5.4, Comsol, Inc., USA). (A) Overall, (B) top, and (C) cross-sectional views of a 3D model consisting of a porous membrane, a microwell, and a working electrode. Boundary conditions and initial values are shown in the illustrations. To simply the model, symmetry was set for the boundary conditions. $\left[\mathrm{Fe}(\mathrm{CN})_{6}\right]^{4-}$ is filled in the model, and its initial value was set to $5 \mathrm{mM}$. The diffusion coefficient was set to $7 \times 10^{-10} \mathrm{~m}^{2} / \mathrm{s}$. Since the sufficient potential is applied to oxidize $\left[\mathrm{Fe}(\mathrm{CN})_{6}\right]^{4^{-}}$to $\left[\mathrm{Fe}(\mathrm{CN})_{6}\right]^{3-}$, the boundary condition of the electrode was set to $0 \mathrm{mM}$. The flux is set to zero at the insulation surface 
and the membrane. In the simulation, the electrochemical system is a reversible oneelectron reaction. The concentration gradient on the electrode, the Faraday constant, the diffusion coefficient, and a unit area were used to calculate the oxidation currents of $\left[\mathrm{Fe}(\mathrm{CN})_{6}\right]^{4-}$. (D) Profile of $\left[\mathrm{Fe}(\mathrm{CN})_{6}\right]^{4-}$ concentration at $150 \mathrm{~s}$ when the gap is $10 \mu \mathrm{m}$. (E) Amperograms when the gap is $1,5,10,20$, and $50 \mu \mathrm{m}$. 
(A)

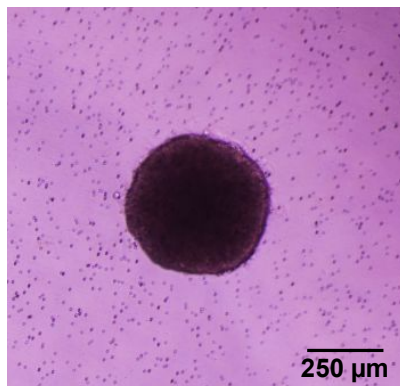

(B)

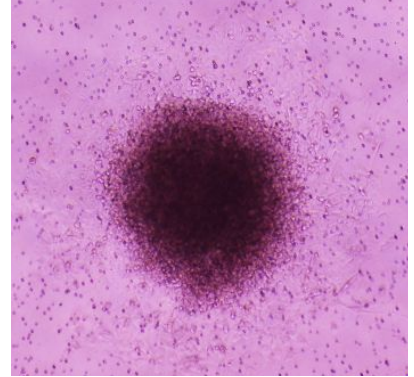

\section{Figure S2}

Phase-contrast images of the HepG2 spheroids on the membrane. (A) Day 1 and (B) day 4. 


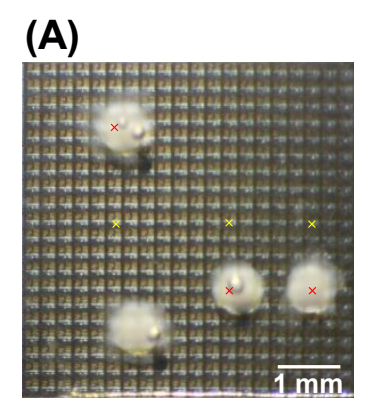

(B)
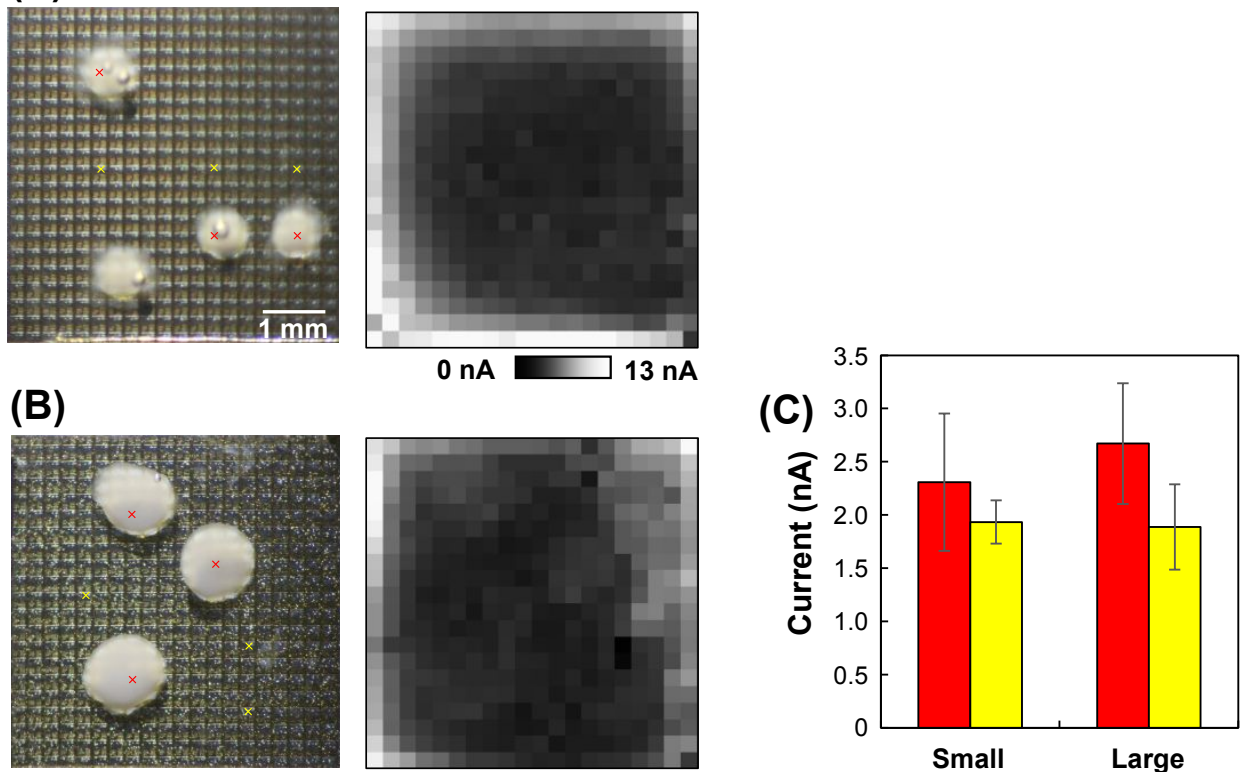

Figure S3

Electrochemical imaging of HUVEC monolayers with large MCF-7 spheroids. (A) Day 1 and (B) day 4. (A, B) Optical (left) and electrochemical images obtained at $299.8 \mathrm{~s}$ (right). (C) Currents from the sensors indicated by red and yellow $\mathrm{x}$ in (A) and (B). (A) is reproduced in Fig. 6. 